\title{
Living Cellular Construct for Increasing the Width of Keratinized Gingiva: Results From a Randomized, Within-Patient, Controlled Trial
}

\author{
Michael K. McGuire, ${ }^{*}$ E. Todd Scheyer, ${ }^{*}$ Marc L. Nevins, ${ }^{\dagger}$ Rodrigo Neiva, ${ }^{\ddagger}$ David L. Cochran, $\S$
} James T. Mellonig, $§$ William V. Giannobile,, and Damien Bates $\|$

Background: The standard of care for increasing keratinized gingiva adjacent to teeth that do not require root coverage is the free gingival graft (FGG). A pilot study indicated that the use of a living cellular construct (LCC) could be effective in this clinical scenario.

Methods: A pivotal, multicenter, randomized, within-patient, controlled, open-label trial was conducted ( $N=96$ patients). After removing the mucosa and keratinized gingiva from the test site, either an LCC or FGG was applied. The primary efficacy endpoint was the ability of the LCC to regenerate $\geq 2 \mathrm{~mm}$ keratinized gingiva at 6 months. Secondary measures were the same color and texture as the adjacent tissue, a 1-mm width of keratinized gingiva at 6 months, patient treatment preference, surgical site sensitivity at 1 week, and patient-reported pain after 3 days. Safety was assessed by reports of adverse events.

Results: At 6 months, the LCC regenerated $\geq 2 \mathrm{~mm}$ of keratinized gingiva in $95.3 \%$ of patients ( 81 of 85 patients; $P<0.001$ versus a $50 \%$ predefined standard). As expected, the FGG generated more keratinized gingiva than the LCC ( $4.57 \pm 1.0 \mathrm{~mm}$ versus $3.2 \pm 1.1 \mathrm{~mm}$, respectively). The gingiva regenerated with the LCC matched the color and texture of the adjacent gingiva. All patients achieved $\geq 1 \mathrm{~mm}$ keratinized gingiva with the LCC treatment by 6 months, and more patients preferred treatment with the LCC than with the FGG. No difference in sensitivity or pain was noted between the treatments. The treatments were well tolerated, and reported adverse events were typical for this type of periodontal surgery.

Conclusion: The use of an LCC may provide a safe and effective therapy for augmenting the zone of keratinized gingiva. J Periodontol 2011;82:1414-1423.

\section{KEY WORDS}

Gingival recession; mouth mucosa; oral surgery; periodontal diseases; regenerative medicine; wound healing.

\footnotetext{
* Private practice, Houston, TX.

$\dagger$ Private practice, Boston, MA.

\# Department of Periodontics, College of Dentistry, University of Florida, Gainesville, FL.

$\S$ Department of Periodontics, University of Texas Health Science Center, San Antonio, TX.

\| Michigan Center for Oral Health Research, University of Michigan School of Dentistry, Ann Arbor, MI.

Il Organogenesis, Canton, MA.
}

$M$ ost clinicians agree that it is preferable to have keratinized gingiva adjacent to teeth. The presence of keratinized gingiva may make homecare more effective, resist frenum pull, and potentially reduce further recession. ${ }^{1,2}$ The absence of an adequate keratinized mucosa has been associated with high plaque accumulation and gingival inflammation. ${ }^{3}$ If a decision is made to increase the zone of the keratinized gingiva, the standard of care for non-rootcoverage techniques has been the free gingival graft (FGG). As a graft, the palatal tissue provides keratinized gingiva, but it retains its palatal phenotype and may be noticeably different in color and texture from the surrounding gingival tissue. Most importantly, the need for a donor site adds to the morbidity (e.g., pain and bleeding) of the procedure for the patient.

A living cellular construct (LCC) comprised of human allogenic fibroblasts, keratinocytes, bovine collagen, and human extracellular matrix proteins has been used for $>10$ years to treat patients with cutaneous wounds, including venous $\mathrm{leg}^{4}$ and diabetic foot ulcers, $, 5,6$ excised burn wounds, ${ }^{7}$ and excisional surgery. ${ }^{8-12}$ Because of the similarities of healing between cutaneous and oral mucosal wounds, it was thought that LCC may deliver better results than the standard FGG in terms of the color and texture

doi: 10.1902/jop.2011.100671 
of the gingiva while providing an adequate amount of new keratinized gingiva.

Although the mechanism of action of the LCC has not been fully elucidated, it has been postulated that it improves the wound environment through growth factor interactions, matrix deposition and degradation, wound coverage, and the provision of responsive cells. ${ }^{13}$ It has been established that LCC does not function as an autograft, ${ }^{14}$ which normally vascularizes, integrates, and persists in situ, retaining the characteristics of the tissue of origin; instead, the LCC appears to stimulate the patient's own cells to regenerate site-appropriate tissue through the modulation and improvement of secondary intention healing. $7,9,10,12,15$ The LCC produces cytokines and cell growth factors involved in tissue development and wound healing (e.g., vascular endothelial growth factor, platelet-derived growth factor, bone morphogenetic protein 2, and transforming growth factor ß). ${ }^{6,16}$ Despite being comprised of allogenic cells, numerous studies $4,5,7-12,14,15,17$ have determined that the LCC does not elicit an immune reaction.

Results from a single-center, randomized, withinpatient, controlled pilot study ${ }^{15}$ with an aim similar to this randomized controlled trial suggested that treatment with the LCC was not statistically significantly different from FGG in terms of recession, clinical attachment level (CAL), bleeding on probing (BOP), resistance to muscle pull, or inflammation. At 6 months, the LCC generated $2.4 \pm 1.0 \mathrm{~mm}$ of keratinized gingiva, without a donor site. However, the LCC provided a statistically significantly better color match and texture than the FGG $(P<0.001)$, and patients preferred the LCC more than FGG $(P=0.041)$. None of the data from the pilot study ${ }^{15}$ was included in the present report.

The present study is designed to determine whether the LCC could achieve a clinically acceptable, safe, and effective amount of keratinized gingiva ( $\geq 2 \mathrm{~mm}$ ) 6 months after surgery in patients who had an insufficient zone of attached gingiva (defined as $<1 \mathrm{~mm}$ attached gingiva) associated with $\geq 2$ non-adjacent teeth. Although $\geq 2 \mathrm{~mm}$ keratinized gingiva (generally corresponding to $1 \mathrm{~mm}$ attached gingiva) has been suggested to be required to maintain gingival health, ${ }^{18}$ no single clinical measures are universally accepted to assess periodontal treatment outcomes. ${ }^{19}$ Thus, we designed a study with one primary and six secondary endpoints that reflected the most commonly used factors that periodontists consider when determining the clinical effectiveness of mucogingival treatment.

\section{MATERIALS AND METHODS}

\section{Patients}

The study protocol was approved by institutional review boards of participating sites (Perio Health
Professionals, Houston, TX; Boston Periodontics and Dental Implants, Boston, Massachusetts; Michigan Center for Oral Research, University of Michigan School of Dentistry, Ann Arbor, MI; and University of Texas Health Science Center, San Antonio, Texas), and all patients gave written informed consent before any study-related procedures were done. Patients were eligible for inclusion in the study if they had: 1) $\geq 18$ and $\leq 70$ years of age, and 2) $\geq 2$ non-adjacent teeth in contralateral quadrants of the same jaw with $\leq 1 \mathrm{~mm}$ attached gingiva that required soft tissue grafting without the need for root coverage. Other inclusion and exclusion criteria are provided in Table 1.

\section{Study Product}

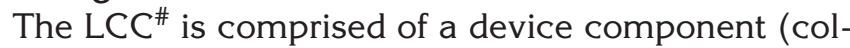
lagen matrix) and biologic components (cells and their products) supplied as a single circular construct $\approx 75 \mathrm{~mm}$ in diameter and $\approx 0.75 \mathrm{~mm}$ thick on a semipermeable polycarbonate membrane on top of an agarose-rich nutrient medium. The LCC was stored at room temperature.

\section{Study Design}

This study was a pivotal, multicenter, randomized, within-patient, controlled, open-label, treatmentcomparison study designed to establish the superiority for predefined efficacy endpoints. After screening, two contralateral teeth were randomly assigned to treatment with the LCC or an FGG as indicated by a predetermined computer-generated randomization scheme. Treatment assignments were stored in sealed envelopes for each patient and opened only at the time of the procedure. As many as three teeth per quadrant were treated, but only one tooth in each quadrant was identified as the study tooth. When two teeth were selected, the study tooth was identified before surgery; when three teeth were selected, the study tooth was the middle tooth. The criteria for selecting target teeth were teeth relatively matched in terms of recession, BOP, width of keratinized tissue, probing depth (PD), and CAL. Baseline measurements were taken for standard periodontal treatment (e.g., plaque score, BOP, PD [using a standardized periodontal probe], keratinized gingiva width, and inflammation). Because of the nature of the control treatment (i.e., FGG), investigators (MKM, ETS, MN, RN, DLC, JTM) and patients could not be masked to the study-tooth treatment assignment or order of surgical procedures. Thus, all clinical measurements were taken by calibrated independent examiners (not the surgeons) at each treatment center in an attempt to reduce the bias. Before study sites began enrolling patients, periodontal examiners were identified and participated in a calibration training session

\footnotetext{
\# CelTx, Organogenesis, Canton, MA.
} 


\section{Table I.}

\section{Inclusion and Exclusion Criteria for Enrollment in the Study}

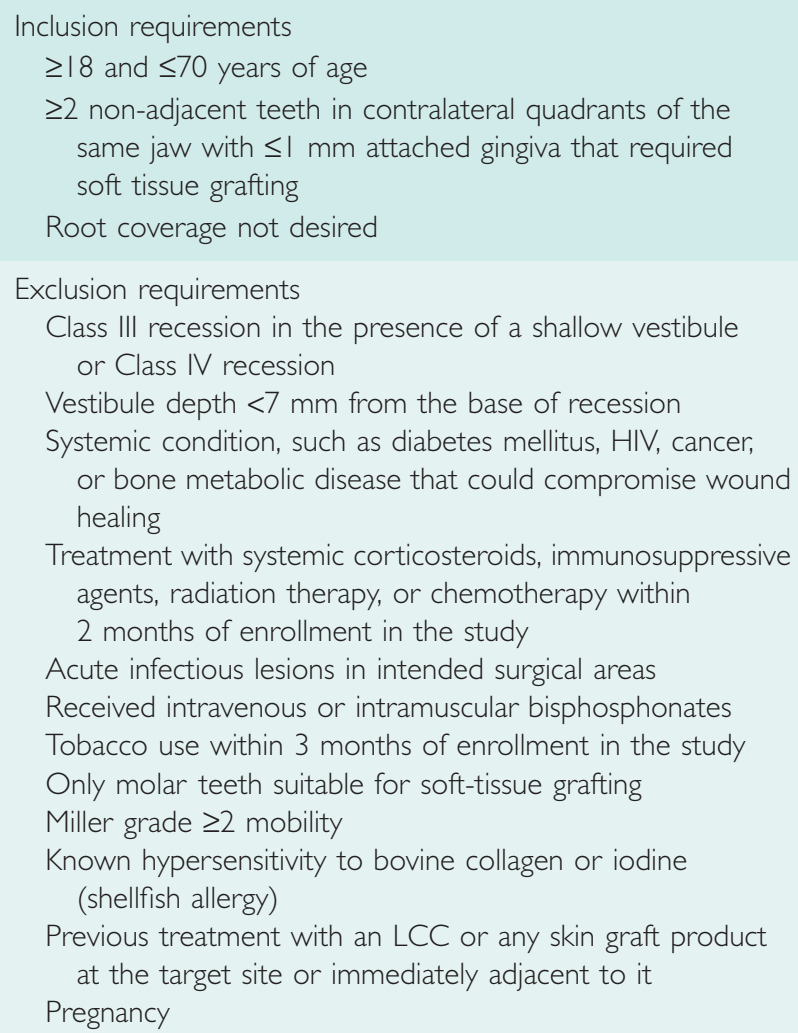

HIV = human immunodeficiency virus.

to evaluate the intra- and interexaminer variability for PD, recession depth, and identification of the mucogingival junction. Levels of both intra- and interexaminer variability were found to be within prespecified limits ( $\kappa \geq 0.7$ for interexaminer comparisons and $\kappa \geq 0.9$ for intraexaminer comparisons).

\section{Surgery}

On day 0 , patients were treated with the LCC on one study tooth and the FGG on the contralateral tooth. Recipient beds were created according to standard procedures that ensured no muscle tension on the bed. ${ }^{20,21}$ The size of the LCC was determined by the investigator and was based upon the number of teeth being treated with the LCC. The LCC was Z-folded (Fig. 1) with minimum dimensions of $6 \mathrm{~mm}$ wide and $10 \mathrm{~mm}$ long. The size of the FGG applied was $4 \mathrm{~mm}$ wide, with the length dictated by the number of teeth being treated. The details of the surgical procedure have been previously reported. ${ }^{15}$ In brief, after the patients received anesthesia with $2 \%$ lidocaine hydrochloride, a partial-thickness dissection was performed to remove any keratinized gingival mucosa and non-keratinized alveolar mucosa from the site.
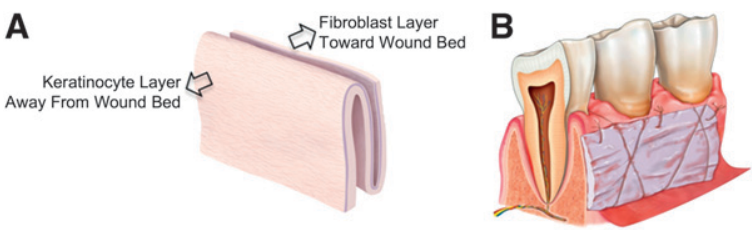

Figure I.

The LCC arranged in a Z-fold fashion (A) and placed over an oral defect in which the mucosal and keratinized gingiva was removed (B). (IA: figure prepared for, and exclusively licensed to, Organogenesis by Mark Lefkowitz; I B: figure prepared for, and exclusively licensed to, Organogenesis by Levent Efe.)

A coronal incision was made at the height of the existing mucosa that extended at least to the line angle of adjacent teeth, and from that point, vertical incisions were made on mesial and distal aspects of study sites to allow $\geq 7 \mathrm{~mm}$ apical to the base of the recession. Mesial and distal incisions were connected apically, and any muscle fibers were removed to create a clean periosteal bed. If feasible, a full-thickness horizontal incision was made just apical to the planned level of the LCC or FGG placement. In this way, the separation of the apical periosteum from the coronal periosteum was accomplished to reduce the risk of the retraction of the treated area during healing.

The graft was harvested from the palatal donor site according to standard practices. ${ }^{21}$ A partial-thickness (i.e., $\approx 1$ to $2 \mathrm{~mm}$ deep) incision was used to harvest a graft the size of the recipient bed. The palatal donor site was covered with surgical dressing** which remained until it fell off on its own or was removed at the 1- or 2-week follow-up visit.

\section{Coverage of Wound Bed}

The LCC was prepared in a Z-fold with the keratinocyte cell layer facing out and was trimmed to the size needed and applied to the wound bed within 15 minutes after removal from the storage bag. The LCC was sutured in place at each papilla (Fig. 1). An additional single layer of the LCC that extended laterally beyond the wound margins was placed over the entire preparation and sutured at the four corners, when possible. The surgical area was placed under tension to confirm that it was free of movement.

The harvested FGG was placed directly on the recipient wound bed and sutured in place using standard FGG techniques. ${ }^{21}$ Both treatment areas were covered with surgical dressing until it fell off on its own or was removed at the 1 - or 2 -week follow-up visit.

\section{Follow-Up Care}

Patients were prescribed antibiotics in accordance with local standards of care and were provided with a $0.12 \%$ solution of chlorhexidine mouthrinse and

* Coe-Pak, GC America, Alsip, IL. 
postoperative oral hygiene instructions. Patients were to contact the investigational site if the surgical dressing fell off before the first follow-up visit (at 1 week). Patients maintained a diary for 1 to 14 days, noting the adherence of the surgical dressing at surgical sites and at the site of the palatal tissue donation, assessing pain, and recording any medications taken for pain.

Follow-up visits occurred at 1 and 4 weeks and 3 and 6 months after surgery. A visit at week 2 was required if the surgical dressing remained at any site at the week- 1 visit. Changes in medications and adverse events were recorded at all visits; photographs of treatment sites were taken, clinical measurements were obtained, and the color and texture of test sites were evaluated. An oral exam was done at 4 weeks and 6 months, and dental prophylaxis was provided at 3 and 6 months. At the 6 -month visit, a patient treatment-preference questionnaire was completed, radiographs of study teeth were taken, and a pregnancy test was given to women of childbearing potential.

\section{Study Endpoints}

The primary efficacy endpoint was the ability of the LCC treatment to regenerate $\geq 2 \mathrm{~mm}$ keratinized gingiva at 6 months. Secondary measures of efficacy were: color the same as the adjacent tissue (visually evaluated), texture (i.e., firmness) the same as adjacent tissue (tactually evaluated), width of keratinized gingiva at 6 months $\geq 1 \mathrm{~mm}$, patient treatment preference which was related to the procedure and outcome, report of surgical site sensitivity (in response to a puff of air) at 1 week, and pain after 3 days. The primary measures of safety included adverse events (overall and mouth-specific and treatment-specific events). Adverse events were reported using a medical dictionary. ${ }^{\dagger \dagger}$ Additional 6-month endpoints that were prospectively measured included the width (in millimeters) of keratinized gingiva and attached gingiva regenerated.

\section{Statistical Analyses}

Statistical analyses were prospectively defined. The power was calculated based on achieving $85 \%$ power for the primary endpoint. A sample size of 74 patients was required to achieve this power. No interim analysis was done. The treatment randomization was determined using a computer-generated randomization scheme that included the type of treatment used on each side and the order of the treatment implementation.

All patients randomly assigned to treatments, treated, and followed for $\geq 1$ week were included in the safety assessment. The first two patients treated by each surgeon (11 patients) were treated for training in material handling and standardization purposes only. Five surgeons completed training with two patients, and one surgeon performed one training case. Data from these patients were included in safety-related analyses but omitted from efficacy analyses. The efficacy population (modified intention-to-treat [mITT] population) included all patients with the exception of those included in the study for training purposes. Continuous variables were summarized using descriptive statistics (i.e., the mean, median, SD, minimum, and maximum) and categoric variables were summarized using frequencies and percentages.

The primary endpoint tested whether $\geq 50 \%$ of patients achieved a $\geq 2$-mm keratinized gingiva threshold at 6 months by using an exact binomial test with a type I error rate of 0.05 . The success threshold of $50 \%$ of patients was prespecified during discussions with the United States Food and Drug Administration, Silver Spring, Maryland. Secondary endpoints were tested using a closed testing strategy in which the order of testing was prespecified. Each test was conducted sequentially at the 0.05 level until a test was found not to be statistically significant. Endpoints for color and texture compared the tissue at the surgical site with the adjacent non-treated tissue (superiority at 6 months). For a band of keratinized gingiva $\geq 1 \mathrm{~mm}$, the endpoint was the superiority versus a prespecified standard empirically chosen of $80 \%$. The patient preference for treatment and endpoints of surgical site sensitivity of mild or absent after 1 week and pain absent after 3 days were superiority tests between treatments. The patient questionnaire was not validated; however, the study staff was trained to ensure the uniform questioning and recording of responses.

\section{RESULTS}

\section{Patients}

The study commenced in October 2007, and the last patient visit was in December 2008. A total of 119 patients at four sites in the United States were screened, with 96 patients randomly assigned and treated (Fig. 2). Of the 96 patients assigned to treatment, 11 patients were considered training patients and included in the safety data analyses only. The numbers of patients enrolled from investigative sites were as follows: Perio Health Professionals $(n=34)$, Boston Periodontics and Dental Implants $(n=30)$, Michigan Center for Oral Research $(n=29)$, and University of Texas Health Science Center $(n=3)$. Consistent with study inclusion criteria, all patients had mucogingival deformities at the time of surgery, with no other clinically remarkable findings upon oral examinations (Table 2). All 96 patients completed the study (to month 6).

\section{Surgery}

The study surgery was completed under appropriate surgical conditions at all sites. The surgical

$\dagger \dagger$ Medical Dictionary for Regulatory Activities (MedDRA) v.10.0 coding program, Cerner Corporation, Kansas City, MO. 


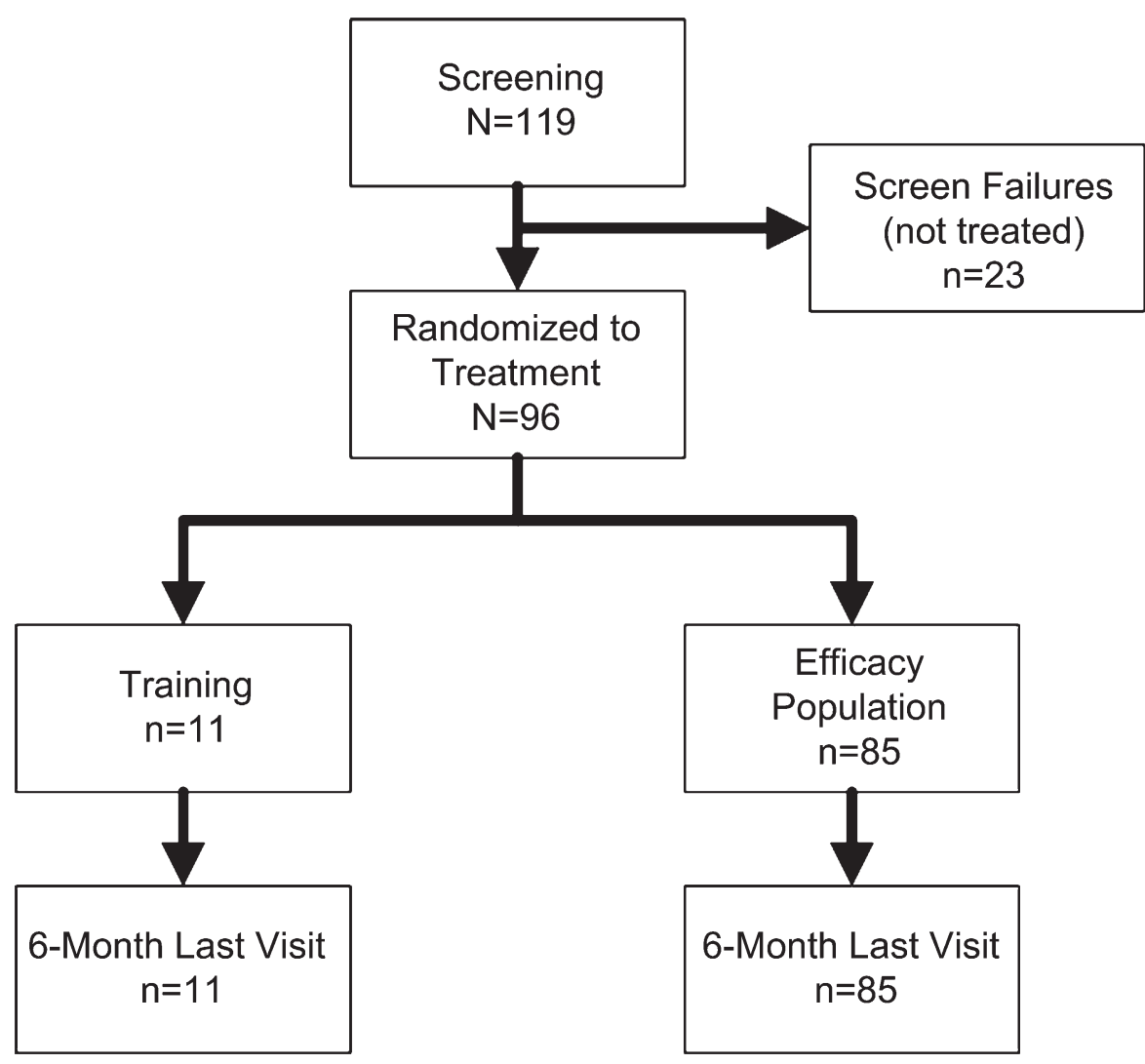

Figure 2.

Disposition of patients.

procedures, including training cases, were performed on the mandible in 89 (93\%) patients, with the LCC being applied to the right side in 50 (52\%) patients. All patients tolerated surgery well. Most surgical procedures used the cemento-enamel junction as the reference point (LCC: 72\%; FGG: 66\%). Mean and median alveolar bone levels and surgical position margins were similar between both sides of the mouth for any given patient. Additional baseline characteristics of surgical sites are provided in Table 3 .

Lengths of palatal grafts ranged from 8 to $30 \mathrm{~mm}$, with widths $\approx 4 \mathrm{~mm}$ in all but two patients who had grafts with widths $\approx 5 \mathrm{~mm}$. Lengths of LCC were similar to those reported for palatal grafts, but widths varied from 5 to $20 \mathrm{~mm}$. No patient required a reapplication of LCC or surgical dressing during the study. In one patient, the palatal graft was taken from the side of the mouth treated with the LCC.

\section{Efficacy}

Regeneration of keratinized gingiva at 6 months. At the 6 -month visit in 81 of 85 (95.3\%) patients, the sites that were treated with the LCC had regenerated a clinically relevant width of keratinized gingiva (i.e., $\geq 2 \mathrm{~mm})$ compared to the $50 \%$ standard $(P<0.001$; exact binomial test; 95\% confidence interval [CI]:
$88.4 \%$ to $98.7 \%)$. As expected, the FGG generated statistically significantly $(P<0.001)$ more keratinized gingiva than the LCC $(4.57 \pm 1.0 \mathrm{~mm}$ versus $3.21 \pm$ $1.1 \mathrm{~mm}$, respectively). The four (4.7\%) patients who did not achieve keratinized gingiva $\geq 2$ $\mathrm{mm}$ achieved a width $\geq 1 \mathrm{~mm}$ (Table 4). All patients in the mITT population achieved $\geq 1 \mathrm{~mm}$ keratinized gingiva with the LCC treatment by 6 months, significantly exceeding the standard established at the beginning of the study, which stipulated that $\geq 80 \%$ of patients should have achieved $\geq 1 \mathrm{~mm}$ keratinized gingiva (LCC: $P<0.001$, exact binomial test; 95\% CI: $95.8 \%$ to $100.0 \%)$. Figure 3 provides a photographic depiction of the results achieved with both treatments.

Although not defined as a primary or secondary endpoint, the amount of attached gingiva generated was captured. At 6 months, sites treated with the LCC generated $1.77 \pm 1.32 \mathrm{~mm}$ attached gingiva, whereas the FGG sites had $3.17 \pm 1.17 \mathrm{~mm}$ attached gingiva.

Color. The color of both treated recipient sites was compared to the adjacent, non-treated gingiva at 4 weeks and 3 and 6 months, and the endpoint assessed whether the color was the same as adjacent tissue at 6 months. At all time points, the gingiva of the tooth treated with the LCC was scored as equally red (i.e., the same) as the adjacent gingiva more often than the gingiva of the tooth treated with the FGG (Table 5). At month 6, a significantly larger proportion of patients treated with the LCC, compared to the FGG, achieved a color that was comparable with the color of the adjacent tissue $(P<0.001$; McNemar test).

Texture. The texture of both treated recipient sites was compared to the adjacent, non-treated tissue at 4 weeks and 3 and 6 months and assessed whether the firmness was equivalent to the adjacent tissue at 6 months. At all time points, the gingiva of the tooth treated with the LCC was scored as equally firm as the adjacent gingiva more often than the gingiva of the tooth treated with the FGG (Table 5). At 6 months, a significantly larger proportion of patients treated with the LCC, compared to the FGG, achieved a texture comparable to the adjacent tissue $(P<0.001$; McNemar test). 
Table 2.

\section{Demographic and Clinical Characteristics of Patients at Baseline by Cohort}

\begin{tabular}{|c|c|c|c|}
\hline Characteristic & Efficacy* $(n=85)$ & Training $(n=||)$ & Total $(n=96)$ \\
\hline \multicolumn{4}{|l|}{$\operatorname{Sex}(n[\%])$} \\
\hline Female & $46(54)$ & $6(55)$ & $52(54)$ \\
\hline Male & $39(46)$ & $5(45)$ & $44(46)$ \\
\hline \multicolumn{4}{|l|}{ Age (years) (n [\%]) } \\
\hline Mean (SD) & $46.9(12.7)$ & $49.4(16.7)$ & $47.1(13.1)$ \\
\hline Median & 48.3 & 53.6 & 48.8 \\
\hline Minimum, maximum & 18.0, 70.8 & $21.2,70.3$ & 18.0, 70.8 \\
\hline \multicolumn{4}{|l|}{ Race (n [\%]) } \\
\hline White & $77(9 \mid)$ & $10(91)$ & $87(91)$ \\
\hline Black & $1(1)$ & $0(0)$ & $1(1)$ \\
\hline Asian & $4(5)$ & I (9) & $5(5)$ \\
\hline Other & $3(3)$ & $0(0)$ & $3(3)$ \\
\hline \multicolumn{4}{|c|}{ Previous tobacco use (n [\%]) } \\
\hline Yes & $34(40)$ & $4(36)$ & $38(40)$ \\
\hline No & $51(60)$ & $7(64)$ & $58(60)$ \\
\hline \multicolumn{4}{|c|}{ Selected dental history $(\mathrm{n}[\%])^{\dagger}$} \\
\hline None & $12(14.1)$ & $0(0)$ & $12(12.5)$ \\
\hline Loose teeth & $14(16.5)$ & $4(36.4)$ & $18(18.8)$ \\
\hline Bleeding gums & $29(34.1)$ & $3(27.3)$ & $32(33.3)$ \\
\hline Grinding/clenching & $42(49.4)$ & $5(45.5)$ & $47(49.0)$ \\
\hline Sores in mouth & $17(20.0)$ & $2(18.2)$ & $19(19.8)$ \\
\hline \multicolumn{4}{|c|}{ Previous periodontal work (n [\%]) } \\
\hline Yes & $58(68)$ & $9(82)$ & $67(70)$ \\
\hline No & $27(32)$ & $2(18)$ & $29(30)$ \\
\hline
\end{tabular}

* Included all patients except those included in study training.

$\uparrow$ Only clinically relevant parameters are presented.

Patient preference. At 6 months, more patients preferred the LCC treatment compared to the FGG treatment $(71.8 \%$ versus $28.2 \%$, respectively; $P<0.001$; 95\% CI: $61.0 \%$ to $81.0 \%)$. At 6 months, more patients in the mITT population preferred the appearance of the LCC compared to the FGG site $(76.5 \%$ versus $23.5 \%$, respectively; $P<0.001$; exact binomial test; $95 \%$ CI: $66.0 \%$ to $85.0 \%$ ).

Surgical site sensitivity. The sensitivity at the palatal graft site and both treatment sites after 1 week was not frequently reported in the study population. No sensitivity was noted in $>70 \%$ of patients at any site after 1 week, and no patient reported a severe sensitivity at any site at any evaluation period. No difference in sensitivity was noted between the LCC site and FGG site (data not shown).

Pain. More than one-half of the patients reported some pain at one of the procedure sites (palatal graft, LCC, or FGG) for the first 6 days of self-assessment, as recorded in daily diaries. Although pain at the treatment site was reported more frequently by patients at the LCC site compared to the FGG site at 3 days
(70.6\% versus $62.3 \%$, respectively) and 7 days (45.9\% versus $37.7 \%$, respectively), differences between groups were not statistically significant. Most of the pain was reported as being mild or moderate; $<5 \%$ of patients at any time point reported pain as severe. Pain was reported at the palatal graft site in $43.6 \%$ of patients on day 1 , and $>35 \%$ of patients continued to report pain at this site on days 2 through 4, with some patients reporting pain at day 12. Other measurements taken at baseline (e.g., plaque score, BOP, PD, and inflammation) were unremarkable (data not shown).

Safety. All treatments were well tolerated in this patient population, and events reported were typical of those in patients undergoing this type of periodontal surgery. Because of the localized application of the LCC, adverse events were summarized by the event location (i.e., LCC, FGG, or palatal graft, mouth, or other; Table 6).

A total of 24 of 96 (25\%) patients reported $\geq 1$ adverse event during the study, with a total of 43 events reported, with most events reported as mild or moderate in severity. No event was reported by $>2$ patients. The most commonly reported events were infections (10 events in eight [8.3\%] patients) and included nasopharyngitis, respiratory tract infection, sinusitis, and upper respiratory tract infection $(\mathrm{n}=2$ each). Eight patients reported nine gastrointestinal events, including two reports each of aphthous stomatitis and dental caries. Three patients reported an adverse event occurring at the LCCtreated site. Two of these events were secondary to the inadvertent placement of the polycarbonate membrane on which the LCC was supplied, and the third was a mouth ulceration. Two patients experienced adverse events occurring at the palatal harvest site (a postprocedural hemorrhage and thrombosis), and two patients experienced adverse events occurring at the FGG-treated site (gingivitis and skin exfoliation).

Three serious adverse events were reported during the study and required hospitalization: one each for 
Table 3.

\section{Selected Baseline Characteristics of Surgical Sites of the mITT Cohort $(n=85)$}

\begin{tabular}{lcc}
\hline Characteristic & LCC & FGG \\
\hline $\begin{array}{l}\text { Presence of plaque (n [\%]) } \\
\text { Buccal }\end{array}$ & $18(2 \mathrm{I})$ & $19(22)$ \\
Lingual & $27(32)$ & $29(34)$ \\
BOP (n [\%]) & $23(27)$ & $18(21)$ \\
PD (mm) & & \\
Mean (SD) & $1.39(0.49)$ & $1.35(0.49)$ \\
Keratinized gingiva & & \\
$\quad$ width (mm) & & \\
Mean (SD) & $1.41(0.72)$ & $1.43(0.69)$ \\
Attached gingiva & & \\
$\quad$ width (mm) & & \\
Mean (SD) & $0.02(0.76)$ & $0.08(0.79)$ \\
Inflammation (n [\%]) & & \\
0 (normal) & $71(83.5)$ & $70(82.4)$ \\
I (mild of any portion of & $12(14.1)$ & $14(16.5)$ \\
$\quad$ the marginal unit) & $2(2.4)$ & $1(1.2)$ \\
2 (mild of the entire & & \\
$\quad$ gingival unit) & $0(0)$ & $0(0)$ \\
3 (moderate) & $0(0)$ & $0(0)$ \\
4 (severe) & & \\
Treatment jaw (n [\%]) & $80(94.1)$ & $80(94.1)$ \\
Mandible & $5(5.9)$ & $5(5.9)$ \\
Maxilla & & \\
\hline
\end{tabular}

Table 4.

\section{Amount of Soft Tissue Regenerated at 6 Months}

\begin{tabular}{lcc}
\hline Tissue Type & LCC & FGG \\
\hline $\begin{array}{c}\text { Keratinized gingiva (mm; } \\
\text { mean [SD] width) }\end{array}$ & $3.21(1.14)$ & $4.57(1.00)$ \\
$\begin{array}{c}\text { Attached gingiva* (mm; } \\
\text { mean [SD]) }\end{array}$ & $1.77(1.32)$ & $3.17(1.17)$ \\
\hline
\end{tabular}

* Prospectively collected but not identified as a primary or secondary endpoint.

pneumonia, chest pain, and metastatic malignant fibrous histiocytoma. The investigator assessed these events to be unrelated or unlikely to be related (in the case of histiocytoma) to the treatment.

\section{DISCUSSION}

The absence of sufficient keratinized gingiva may lead to gingival recession, which in turn may result in the loss of a portion of the cortical plate and a worsen- ing prognosis for the integrity of the affected tooth. Although a meta-analysis suggested that no single clinical measure was universally acceptable to assess periodontal treatment outcomes, ${ }^{19}$ the objective standard of $\geq 2 \mathrm{~mm}$ keratinized gingiva in mucogingival procedures is generally accepted. In this study, we evaluate the utility of an LCC to regenerate keratinized gingiva when applied to a wound bed in which the mucosa or keratinized gingiva are removed. The use of the LCC gave a $95.3 \%$ success rate in the primary effectiveness endpoint (e.g., $\geq 2 \mathrm{~mm}$ keratinized gingiva at month 6) $(P<0.001$ versus 50\% standard), and all patients achieved $\geq 1 \mathrm{~mm}$ keratinized gingiva ( $P<0.001$ versus $80 \%$ standard). The clinical significance of these findings was supported by the fact that a mean of $1.77 \pm 1.32 \mathrm{~mm}$ attached gingiva was present at 6 months. Other endpoints, the color and texture compared to adjacent tissue and patient preferences, were statistically significant in favor of the LCC ( $P<0.001$ for all measures).

Statistically significant differences were not obtained for the secondary efficacy endpoints of the absence of pain by 3 days and a minimal surgical sensitivity at the week-1 time point. The harvesting of the FGG from the palate was associated with some morbidity; however, the within-patient study design may not have allowed for the accurate localization of pain and sensitivity because of multiple surgical sites within the mouth. It was also possible that the bed preparation for LCC was somewhat larger than the bed for FGG, which could have influenced painrelated endpoints.

Even though FGG yielded statistically significantly more keratinized tissue than LCC $(4.57 \pm 1.0 \mathrm{~mm})$, the use of LCC may have several advantages compared to the use of FGG. First, a second surgical site, the palatal donor-site, is not required with its use, eliminating pain and bleeding at the donor site. Second, an unlimited supply of readily available material may prevent undertreatment in situations of limited autogenous tissue. The use of LCC provides an unlimited amount of material to treat demanding cases in one surgical visit. Furthermore, based on the unlimited availability of the LCC, the augmentation of keratinized gingiva could be indicated for extensive oral rehabilitation cases for which the palatal tissue would be insufficient. Third, the LCC is not a graft but affects healing by improving the rate and quality of wound repair by a secondary intention ${ }^{10,14}$ and, therefore, allows the regeneration of a clinically relevant width of site-appropriate (form and function) tissue that has the same color and texture of surrounding tissues. Although palatal tissue is a reliable means to generate additional keratinized gingiva, the tissue remains palatal, not gingival, and is often paler and of different texture than surrounding tissue. Shrinkage is often 


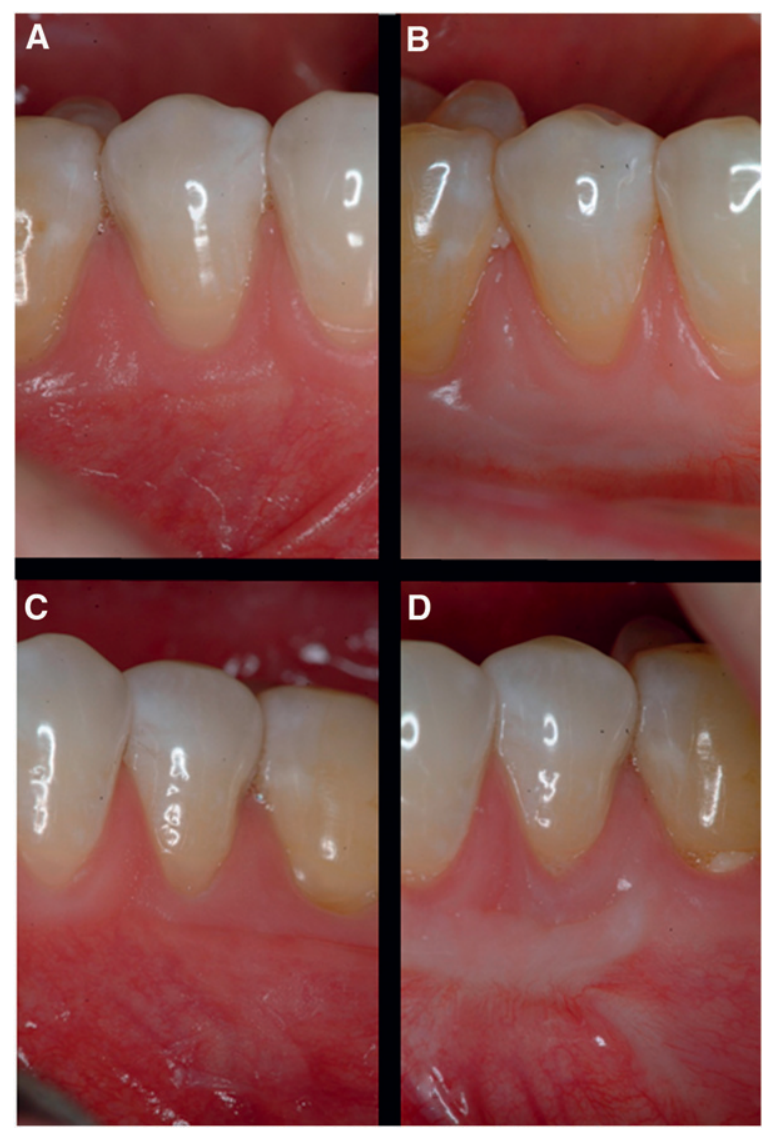

Figure 3.

Photographs of treatment sites within a single patient taken at baseline $(\boldsymbol{A}$ and $\mathbf{C})$ and 6 months after the application of LCC (B) or FGG (D).

seen with an FGG, and overcorrection is sometimes needed, requiring a larger donor site with its inherent morbidities. ${ }^{22,23}$ Because the LCC does not function as a graft, and the cells of the device do not persist, it made no sense to standardize the width of LCC to the width of FGG. An effort was made to place as wide a piece of the LCC as the vestibule would allow. Patients with shallow vestibules may not be good candidates for this procedure.

The results from this study extend the findings from a pilot investigation. ${ }^{15}$ However, changes from the pilot study included the definition of a minimum size for the Z-folded LCC of $5 \times 10 \mathrm{~mm}$, with deeper vestibules permitted to have a wider treatment area. In an attempt to protect the LCC, an additional single layer of LCC was applied, which was not used in the pilot study. The FGG with a 4-mm width was selected to account for interpatient anatomic variability, which sometimes limits the width of harvested palatal tissue. ${ }^{21}$ Additionally, the 4 -mm width was used, compared to a larger width of the FGG, to minimize the chance of a poor esthetic outcome (color and texture match) commonly seen with FGG proce-
Table 5.

Measure (n [\%]) of Color and Texture Compared With Tissue Adjacent to the Surgical Site in the mITT Cohort $(n=85)$ at 4 Weeks and 3 and 6 Months

\begin{tabular}{lcc}
\hline Study Visit & LCC & FGG \\
\hline Color & & \\
4 weeks & & \\
More red & $28(32.9)$ & $6(7.1)$ \\
Equally red & $56(65.9)$ & $4 I(48.2)$ \\
Less red & $1(1.2)$ & $38(44.7)$ \\
3 months & & \\
More red & $3(3.5)$ & $1(1.2)$ \\
Equally red & $80(94.1)$ & $29(34.1)$ \\
Less red & $2(2.4)$ & $55(64.7)$ \\
6 months & & \\
More red & $4(4.7)$ & $0(0)$ \\
Equally red & $79(92.9)$ & $23(27.1)$ \\
Less red & $2(2.4)$ & $62(72.9)$ \\
Texture & & \\
4 weeks & & \\
More firm & $2(2.4)$ & $27(31.8)$ \\
Equally firm & $63(74.1)$ & $57(67.1)$ \\
Less firm & $20(23.5)$ & $1(1.2)$ \\
3 months & & \\
More firm & $0(0)$ & $37(43.5)$ \\
Equally firm & $80(94.1)$ & $48(56.5)$ \\
Less firm & $5(5.9)$ & $0(0)$ \\
6 months & $0(0)$ & $39(45.9)$ \\
More firm & $81(95.3)$ & $46(54.1)$ \\
Equally firm & $4(4.7)$ & $0(0)$ \\
Less firm & \\
\hline
\end{tabular}

All patients had $\geq 1$ tooth treated with each therapy.

dures while reliably generating a clinically relevant amount (i.e., $\geq 2 \mathrm{~mm}$ ) of keratinized gingiva.

Nevertheless, the pilot study results showed the generation of $2.4 \pm 1.02 \mathrm{~mm}$ of keratinized gingiva, whereas the present study results showed the generation of $3.2 \pm 1.14 \mathrm{~mm}$ of keratinized gingiva. This amount of keratinized gingiva exceeded the proposed minimum keratinized gingiva necessary to maintain gingival health by $>1 \mathrm{~mm} .{ }^{18}$ Eighty-one of 85 (95.3\%) patients treated with the LCC regenerated a clinically relevant width of keratinized gingiva (i.e., $\geq 2 \mathrm{~mm}$ ).

As with any study, the interpretation of these results was limited by certain aspects of the study design. Although the duration of this study was the typical length for soft tissue studies, ${ }^{24-26}$ a longer follow-up would have allowed us to more thoroughly evaluate the durability of the tissue regenerated in the presence of the LCC and whether further creeping attachment occurred with time. ${ }^{27}$ Additional studies need to be conducted to determine whether patients 
Table 6.

\section{Overall Incidence of Adverse Events by Location, Severity, and Relationship to Treatment by Patients Reporting $\geq 1$ Event (Safety Population, $\mathrm{n}=96$ )}

\begin{tabular}{|c|c|c|c|c|c|}
\hline Adverse Events & LCC & FGG & Donor Site & Mouth & Other \\
\hline $\begin{array}{c}\text { Overall (n [\%]) } \\
\text { By severity } \\
\text { (n [\%]) }\end{array}$ & $3(3.1)$ & $2(2.1)$ & $2(2.1)$ & $5(5.2)$ & $17(17.7)$ \\
\hline Mild & $3(3.1)$ & I (1.0) & I (1.0) & $4(4.2)$ & $14(14.6)$ \\
\hline Moderate & 0 & 0 & I (1.0) & 0 & $5(5.2)$ \\
\hline $\begin{array}{c}\text { Severe } \\
\text { By relationship } \\
(\mathrm{n}[\%])\end{array}$ & 0 & I (1.0) & 0 & I (I.0) & $2(2.1)$ \\
\hline Not related & I (I.0) & I (1.0) & $2(2.1)$ & $3(3.1)$ & $14(14.6)$ \\
\hline Related* & $2(2.1)$ & I (1.0) & 0 & $2(2.1)$ & $3(3.1)$ \\
\hline
\end{tabular}

* Includes unlikely, possible, probable, and definite.

find the benefit of not having a donor site offsets the additional cost of the tissue-engineered device. Additionally, because of the nature of the surgical procedures, we were unable to mask the investigators, and although we do not believe this to be a substantial influence, some bias in the performance of the procedures may have occurred. ${ }^{28}$ Last, the use of an autograft may not have been the best control to evaluate a treatment for which the mode of healing was via a secondary intention. However, a more appropriate control procedure, such as the "push back technique" 29 or a gingivectomy, ${ }^{30}$ is no longer considered a viable treatment option.

\section{CONCLUSIONS}

The results of this study suggest that LCC may be a safe and effective alternative to the standard of care, FGG, for restoring gingival tissue adjacent to teeth that do not require root coverage. The treatments were well tolerated, and the adverse events reported were events typical of this type of surgery. These positive results open the possibility of considering LCC as a treatment for oral mucosal surface defects for which the regeneration of site-appropriate tissue is required.

\section{ACKNOWLEDGMENTS}

This human clinical trial was registered at clinicaltrials.gov as NCT00587834. The study was sponsored by Organogenesis, Canton, Massachusetts, and the National Institutes of Health/National Center for Research Resources, Bethesda, Maryland (UL 1RR024986). MedDRA ${ }^{\circledR}$ is a registered trademark of the International Federation of Pharmaceutical Manufacturers and Associations (IFPMA). Drs. Tae-ju
Oh and Thiago Santos Morelli, Department of Periodontics, University of Michigan, contributed to the clinical aspects of the investigation. The following individuals served as calibrated examiners and performed clinical measurements and assessments: Rebecca Garcia, registered dental hygienist, Perio Health Professionals; Dr. Richard Finlayson, Department of Periodontics, University of Texas Health Science Center; Dr. Tae-Ju Oh, Michigan Center for Oral Health Research, University of Michigan School of Dentistry; and Cillen Cosentino, registered dental hygienist, Boston Periodontics and Dental Implants. MaryAnn Foote, MA Foote Associates, Westlake Village, California, was remunerated by Organogenesis for assisting in the preparation of the manuscript. Katherine Giovino, director, clinical operations; Susan Routhier, clinical program manager; and Phillip Golden, senior clinical research associate, Organogenesis, were instrumental in the management of the clinical trial. Organogenesis manufactured the LCC used in this study. Statistical analyses were performed by Averion International, Southborough, Massachusetts. Dr. McGuire is a consultant for Organogenesis and received consulting fees and honoraria for lectures from Organogenesis; Dr. Cochran received a research grant and consulting fees from Organogenesis; Dr. Giannobile served on the scientific advisory board for Organogenesis; and Dr. Bates is chief medical officer and a shareholder of Organogenesis. Drs. Scheyer, Nevins, Neiva, and Mellonig report no financial relationships related to any products involved in this study.

\section{REFERENCES}

1. Wennström J, Lindhe J. Role of attached gingiva for maintenance of periodontal health. Healing following excisional and grafting procedures in dogs. J Clin Periodontol 1983;10:206-221.

2. Nevins $M$. Attached gingiva-mucogingival therapy and restorative dentistry. Int J Periodontics Restorative Dent 1986;6(4):9-27.

3. Chung DM, Oh TJ, Shotwell JL, Misch CE, Wang HL. Significance of keratinized mucosa in maintenance of dental implants with different surfaces. J Periodontol 2006;77:1410-1420.

4. Falanga V, Margolis D, Alvarez O, et al. Rapid healing of venous ulcers and lack of clinical rejection with an allogeneic cultured human skin equivalent. Arch Dermatol 1998;134:293-300.

5. Veves A, Falanga V, Armstrong DG, Sabolinski ML; Apligraf Diabetic Foot Ulcer Study. Graftskin, a human skin equivalent, is effective in the management of noninfected neuropathic diabetic foot ulcers: A prospective randomized multicenter clinical trial. Diabetes Care 2001;24:290-295.

6. Brem H, Young J, Tomic-Canic M, Isaacs C, Ehrlich HP. Clinical efficacy and mechanism of bilayered living human skin equivalent (HSE) in treatment of diabetic foot ulcers. Surg Technol Int 2003;11: 23-31. 
7. Waymack P, Duff RG, Sabolinski M; The Apligraf Burn Study Group. The effect of a tissue engineered bilayered living skin analog, over meshed split-thickness autografts on the healing of excised burn wounds. Burns 2000;26:609-619.

8. Eaglstein WH, Alvarez OM, Auletta M, et al. Acute excisional wounds treated with a tissue-engineered skin (Apligraf). Dermatol Surg 1999;25:195-201.

9. Muhart M, McFalls S, Kirsner RS, et al. Behavior of tissue-engineered skin: A comparison of a living skin equivalent, autograft, and occlusive dressing in human donor sites. Arch Dermatol 1999;135:913-918.

10. Gohari S, Gambla C, Healey M, et al. Evaluation of tissueengineered skin (human skin substitute) and secondary intention healing in the treatment of full thickness wounds after Mohs micrographic or excisional surgery. Dermatol Surg 2002;28:1107-1114, discussion 1114.

11. Donohue KG, Carson P, Iriondo M, et al. Safety and efficacy of a bilayered skin construct in full-thickness surgical wounds. J Dermatol 2005;32:626-631.

12. Hu S, Kirsner RS, Falanga V, Phillips T, Eaglstein WH. Evaluation of Apligraf persistence and basement membrane restoration in donor site wounds: A pilot study. Wound Repair Regen 2006;14:427-433.

13. Morelli T, Neiva R, Nevins ML, et al. Angiogenic biomarkers and healing of living cellular constructs [published online ahead of print Jan 19, 2011]. J Dent Res doi: 10.1177/0022034510389334.

14. Griffiths M, Ojeh N, Livingstone R, Price R, Navsaria H. Survival of Apligraf in acute human wounds. Tissue Eng 2004; 10:1180-1195.

15. McGuire MK, Scheyer ET, Nunn ME, Lavin PT. A pilot study to evaluate a tissue-engineered bilayered cell therapy as an alternative to tissue from the palate. $J$ Periodontol 2008;79:1847-1856.

16. Milstone LM, Asgari MM, Schwartz PM, Hardin-Young J. Growth factor expression, healing, and structural characteristics of Graftskin (Apligraf). Wounds 2000; 12(Suppl. 5A):12A-19A.

17. Wilkins LM, Watson SR, Prosky SJ, Meunier SF, Parenteau NL. Development of a bilayered living skin construct for clinical applications. Biotechnol Bioeng 1994;43:747-756.

18. Lang NP, Löe H. The relationship between the width of keratinized gingiva and gingival health. J Periodontol 1972;43:623-627.

19. Ryan ME. Clinical attachment level change as an outcome measure for therapies that slow the progression of periodontal disease. J Int Acad Periodontol 2005;7(Suppl. 4):162-171; discussion 172-174.

20. Miller PD Jr. Root coverage using the free soft tissue autograft following citric acid application. III. A successful and predictable procedure in areas of deepwide recession. Int $J$ Periodontics Restorative Dent 1985;5(2):14-37.
21. Takei HH, Azzi RR, Han TJ. Periodontal plastic and esthetic surgery. In: Newman MG, Takei HH, Klokkevold PR, Carranza RA, eds. Carranza's Clinical Periodontology, 10th ed. St. Louis, MO: Saunders/Elsevier; 2006: 1005-1029.

22. Orsini M, Orsini G, Benlloch D, Aranda JJ, Lázaro P, Sanz M. Esthetic and dimensional evaluation of free connective tissue grafts in prosthetically treated patients: A 1 -year clinical study. J Periodontol 2004;75: 470-477.

23. Hatipoğlu H, Keçeli HG, Güncü GN, Sengün D, Tözüm TF. Vertical and horizontal dimensional evaluation of free gingival grafts in the anterior mandible: A case report series. Clin Oral Investig 2007;11:107-113.

24. Cardaropoli D, Cardaropoli G. Healing of gingival recessions using a collagen membrane with a hemineralized xenograft: A randomized controlled clinical trial. Int J Periodontics Restorative Dent 2009;29:59-67.

25. Santamaria MP, Ambrosano GM, Casati MZ, Nociti FH Jr., Sallum AW, Sallum EA. Connective tissue graft plus resin-modified glass ionomer restoration for the treatment of gingival recession associated with noncarious cervical lesion: A randomized-controlled clinical trial. J Clin Periodontol 2009;36:791-798.

26. Sanz M, Lorenzo R, Aranda JJ, Martin C, Orsini M. Clinical evaluation of a new collagen matrix (Mucograft prototype) to enhance the width of keratinized tissue in patients with fixed prosthetic restorations: A randomized prospective clinical trial. J Clin Periodontol 2009;36:868-876.

27. Agudio G, Nieri M, Rotundo R, Franceschi D, Cortellini P, Pini Prato GP. Periodontal conditions of sites treated with gingival-augmentation surgery compared to untreated contralateral homologous sites: A 10- to 27-year long-term study. J Periodontol 2009;80: 1399-1405.

28. Chambrone L, Sukekava F, Araújo MG, Pustiglioni FE, Chambrone LA, Lima LA. Root-coverage procedures for the treatment of localized recession-type defects: A Cochrane systematic review. J Periodontol 2010;81: 452-478.

29. Ariaudo AA, Tyrrell HA. Repositioning and increasing the zone of attached gingiva. J Periodontol 1957;28: 106-110.

30. Wennström J. Regeneration of gingiva following surgical excision. A clinical study. J Clin Periodontol 1983; 10:287-297.

Correspondence: Dr. Michael K. McGuire, Private practice, 3400 S. Gessner, \#102, Houston, TX 77063. E-mail: mkmperio.swbell.net.

Submitted November 9, 2010; accepted for publication February 1, 2011. 